\title{
Vue d'ensemble sur l'évolution des forces de maintien de l'ordre en Europe : les répercussions de l'européanisation sur la police britannique
}

\author{
Paul Swallow
}

\section{(2) OpenEdition Journals}

Édition électronique

URL : http://journals.openedition.org/conflits/903

DOI : $10.4000 /$ conflits.903

ISSN : 1777-5345

Éditeur :

CCLS - Centre d'études sur les conflits lilberté et sécurité, L'Harmattan

Édition imprimée

Date de publication : 1 décembre 2002

ISBN : 2-7475-4291-2

ISSN : $1157-996 \mathrm{X}$

Référence électronique

Paul Swallow, «Vue d'ensemble sur l'évolution des forces de maintien de l'ordre en Europe : les répercussions de l'européanisation sur la police britannique », Cultures \& Conflits [En ligne], 48 | hiver 2002, mis en ligne le 27 avril 2003, consulté le 30 mars 2021. URL : http://journals.openedition.org/ conflits/903 ; DOI : https://doi.org/10.4000/conflits.903

Ce document a été généré automatiquement le 30 mars 2021.

Creative Commons License 


\title{
Vue d'ensemble sur l'évolution des forces de maintien de l'ordre en Europe : les répercussions de l'européanisation sur la police britannique
}

\author{
Paul Swallow
}

1 «Il y a de cela bien longtemps, lorsque les gens passaient toute leur vie au même endroit, le système de police local suffisait largement à assurer le bien-être de la communauté. Aujourd'hui, ce n'est plus le cas. Un homme qui se trouvait dans le sud de l'Angleterre le matin peut être dans le nord, en Ecosse, le soir même, et s'il s'agit d'un criminel il doit être possible de le retrouver facilement et de le présenter à la justice. Pour cela, il faut qu'il y ait une coordination avec l'autorité centrale. Toute frontière est source de fragilité et de danger. Plus il y a de frontières... plus la fragilité est grande et le danger important $»^{1}$.

2 Sir John Anderson s'exprimait en ces termes en 1923 lors d'une conférence du Chief Constables Club, l'ancêtre de l'actuel syndicat officiel des chefs de police britanniques, l'Association of Chief Police Officers (ACPO) ${ }^{2}$ Sir John Anderson abordait la question des défis auxquels serait confrontée la police britannique qui était alors fragmentée, essentiellement locale et qui manquait de coordination, face à l'évolution du monde moderne, notamment l'avènement de l'automobile, le téléphone, la mobilité des criminels et les besoins de normalisation et de coopération. Ses conseils ont-ils été suivis? Si Sir John avait pu lire le rapport intitulé « International, National and InterForce Crime - the Phillips Report » (Criminalité internationale, nationale et inter-force - le rapport Phillips) commandité par l'ACPO en 1996, il aurait bien été forcé de constater que, soixante-trois ans plus tard, les problèmes de coordination demeurent ${ }^{3}$. Si le Royaume-Uni a peu progressé en matière de coopération interne au cours des soixante dernières années, comment va-t-il faire face à la coopération policière internationale? 
3 Dans le présent article nous exposerons brièvement l'évolution et la structure de la police britannique pour mieux comprendre l'attitude et les réactions de la GrandeBretagne à l'égard des forces publiques européennes. Nous décrirons également l'évolution des aspects policiers des principaux instruments d'unité européenne et de coopération européenne du point de vue britannique et nous finirons en abordant la question des répercussions de l'«européanisation» sur les services de police britanniques.

Organisation actuelle de la police britannique

4 La police britannique telle qu'elle se présente aujourd'hui est le résultat d'un processus d'évolution graduelle. Bien qu'on puisse faire remonter les origines du concept de police au Moyen-Âge, il est permis de penser que la police moderne est née à Glasgow en 1814 avant de s'étendre à Londres en 1829 avec la création de la principale force de police de la capitale britannique, le Metropolitan Police Service. Des unités de police ont été progressivement créées dans tout le pays au cours des cinquante années suivantes, chaque comté ou ville mettant sur pied sa propre force de police en s'appuyant sur les précédents institués dans d'autres parties du pays et en les adaptant en fonction des circonstances qui lui étaient propres. Ce développement essentiellement local a empêché la constitution d'un service de police national en Grande-Bretagne.

5 Vers 1918 le Royaume-Uni comptait environ 188 forces de police séparées mais au fil des ans un lent processus d'unification a eu lieu, l'exemple le plus récent étant la formation en 1964 de la Thames Valley Police (police de la vallée de la Tamise) à partir de cinq unités de police plus petites. Aujourd'hui, il y a 52 forces de police territoriale, ou forces de police «du ministère de l'Intérieur» et toute une série de forces "privées» telles que la British Transport Police (police britannique des chemins de fer), la United Kingdom Atomic Energy Authority Police (service de police du RoyaumeUni rattachée à l'équivalent du Commissariat à l'énergie atomique) et la Royal Parks Constabulary (police des parcs royaux) qui exercent une série de fonctions spécifiques ou spécialisées. La force de police la plus importante est le Metropolitan Police Service de Londres qui compte environ 25000 policiers et les plus petites sont, me semble-t-il, la Royal Botanical Gardens Constabulary (police des jardins botaniques royaux) de Kew Gardens, près de Londres, et la police des jardins royaux d'Edimbourg et du Sussex qui compte environ 15 policiers.

6 Par ailleurs, le Royaume-Uni compte plusieurs agences non policières exerçant des fonctions de « maintien de l'ordre » qui, ailleurs en Europe, seraient souvent du ressort de certaines branches de la police nationale. C'est notamment le cas des services de sécurité (le Security Service) et des services secrets de renseignement (le Secret Intelligence Service), mieux connus respectivement sous le nom de MI5 et MI6, ainsi que du service de l'immigration du Royaume-Uni (le United Kingdom Immigration Service) ${ }^{4}$ et des services douaniers (Her Majesty's Customs and Excise Service - HMCE). L'existence même de certains de ces organismes s'explique par le développement progressif de la police britannique. Par exemple, lorsque l'Etat britannique a eu besoin d'un service de sécurité, aucun organisme policier ne semblait à même d'accomplir cette tâche. Il a donc mis en place un organisme pendant non policier chargé de ces questions. Par conséquent, toute description de la «police britannique» doit tenir compte de toutes ou partie des forces ou agences de police mentionnées ci-dessus. 
7 Par rapport à d'autres pays européens la police du Royaume-Uni présente plusieurs différences majeures et le moment semble venu d'en citer quelques-unes. Pour commencer, au Royaume-Uni les policiers sont des civils et la plupart d'entre eux ne sont pas armés ${ }^{5}$. Il n'y a pas d'équivalent de la Gendarmerie nationale française ou de la Guardia Civil espagnole mais l'armée britannique, par exemple, a sa propre force de police. En fait, il s'agit de deux forces de police: la Royal Military Police (police militaire royale), un régiment militaire constitué de soldats, et la Ministry of Defence Police (police du ministère de la Défense) qui est composée de policiers civils.

8 Par ailleurs, les directeurs de police britanniques se disent indépendants de tout contrôle extérieur (c'est-à-dire du pouvoir politique). Selon Reiner, ils « revendiquent un mandat juridique et moral leur permettant d'agir selon leur conscience $»^{6}$. Cette indépendance s'accompagne d'une relative liberté vis-à-vis du contrôle judiciaire pendant la durée des enquêtes. Par exemple, la police est libre d'enquêter comme elle l'entend sur toutes les affaires ou tous les litiges qui lui semblent appropriés et, jusqu'en 1985, elle pouvait engager toutes les poursuites qu'elle souhaitait. Dans les années 1970, alors que j'étais un jeune gardien de la paix, j'ai enquêté et porté devant les Magistrates' Courts (juridiction inférieure - équivalent du tribunal d'instance) des affaires mineures sans aucune formation juridique ou sans faire appel à la compétence d'un juriste. Or, en 1985, le gouvernement a créé le Crown Prosecution Service (CPS - équivalent du Ministère public) qui permet d'intenter des poursuites au nom de l'État mais ce service n'enquête pas et il n'est pas autorisé à demander à la police de le faire. Aujourd'hui, la police doit soumettre les conclusions de toutes ses enquêtes au CPS qui décide s'il y a suffisamment de preuves à charge pour intenter une action en justice. La police conserve néanmoins le droit d'enquêter en toute indépendance.

Malgré la tradition de fragmentation de la police britannique, un besoin de coordination des services à l'échelle nationale se dessine. Cette évolution est relativement récente et elle s'articule autour de trois grands axes. Le premier est celui des deux principales agences « de police » ayant des responsabilités nationales dans la lutte contre la délinquance, à savoir le service national de renseignements criminels (le National Criminal Intelligence Service - NCIS), qui traite les renseignements portant sur la grande criminalité, et la brigade criminelle nationale (National Crime Squad - NCS) chargée des enquêtes criminelles sur la "grande criminalité organisée ». Et sans que ceci soit spécifié, $75 \%$ des activités de la NCS visent le trafic de drogue. Il y a ensuite l'ACPO qui a une fonction de conseil et de représentation de la police britannique au sens large auprès du gouvernement et enfin les trois associations du personnel : la Chief Police Officers Staff Association (CPOSA) qui représente les chefs de police, la Superintendent's Association qui représente les commissaires et la Police Federation.

Le National Crime Intelligence Service (NCIS) est le plus éminent des organismes policiers à l'échelle nationale. Avant son lancement, en 1992, le besoin de coordination nationale des renseignements avait déjà entraîné plusieurs initiatives ad hoc de moindre envergure, toutes au siège du Metropolitan Police Service au New Scotland Yard. En effet, compte tenu de l'absence de financement ou d'organisation à l'échelle centrale, cette force (de loin la plus importante du Royaume-Uni) était la mieux à même de prendre en charge les frais induits. Les initiatives en question étaient la création d'un office central pour la répression du trafic illicite des stupéfiants (la National Drugs Intelligence Unit), de bureaux régionaux des renseignements criminels (les Regional Criminal Intelligence Offices) et de bureaux chargés de l'hooliganisme 
dans le football, de la fausse monnaie, de la corruption dans le secteur public et de la pédophilie. Le New Scotland Yard a également accueilli le bureau central national (BCN) d'Interpol pour le Royaume-Uni. La présence de ces unités nationales au sein du Metropolitan Police Service a largement contribué à la réputation internationale du New Scotland Yard mais a également provoqué un certain ressentiment dans les autres unités de police en raison de la prééminence de la police londonienne sur la scène mondiale.

11 Le NCIS a été créé le 1er avril 1992 et son siège social ${ }^{7}$ se trouve à Londres, à quelques centaines de mètres du New Scotland Yard. Le NCIS a également prévu la création de cinq bureaux régionaux en s'inspirant des anciens bureaux régionaux des renseignements criminels. Le NCIS emploie environ 600 personnes, dont 300 au siège social londonien. Compte tenu de la diversité de ses tâches, le personnel comprend des représentants de plusieurs des différentes agences qui font un travail de "police » au Royaume-Uni, en particulier du service des douanes (HMCE).

12 Le National Crime Squad (NCS) est la première force nationale de police ayant des pouvoirs opérationnels. Elle est née en avril 1998 de la fusion des anciennes brigades criminelles régionales et comprend uniquement des policiers détachés par leurs forces locales. Elle a pour mission d'enquêter sur le grand banditisme.

13 L'Association of Chief Police Officers (ACPO) ${ }^{8} \mathrm{~d}^{\prime}$ Angleterre, du pays de Galles et d'Irlande du Nord est probablement le plus important organisme policier du pays. Elle représente les quelque 270 officiers ayant rang, au moins, d'Assistant Chief Constable des forces de police créées aux termes de la loi sur la réforme de la police (Police Act) de 1964. Le principal objectif de l'ACPO est de promouvoir l'efficacité, le bon fonctionnement et les intérêts professionnels des services de police en Angleterre, au pays de Galles et en Irlande du Nord. Cela justifie le versement d'une contribution financière gouvernementale directe par l'intermédiaire d'un fonds commun, le Common Police Services Fund, pour aider à doter la police en personnel d'encadrement. En 1999, cette contribution financière a avoisiné les 500000 livres sterling.

14 Pendant de nombreuses années, l'ACPO a endossé le rôle de premier conseiller du gouvernement en matière de maintien de l'ordre. Pour ce faire, l'association a mis en place six comités principaux chargés de questions telles que le crime, les ressources humaines et la formation, la recherche, le terrorisme et le trafic illicite. Elle a également mis sur pied des comités de coordination composés de représentants des six autres comités mentionnés ci-dessus. L'efficacité de l'ACPO en la matière a été contestée ${ }^{9}$ en raison de ses ressources limitées. Outre la gestion de leur poste de police, les directeurs de police ont un lourd cahier des charges mais ils disposent de faibles moyens.

15 Auparavant, l'ACPO était essentiellement une association du personnel mais son rôle de conseil a pris de telles proportions qu'en août 1996 l'association s'est divisée en deux entités: une nouvelle ACPO, chargée d'élaborer des politiques et de conseiller le gouvernement, et la Chief Police Officers' Staff Association (CPOSA - association du personnel des chefs de police). Selon son secrétaire général, la première a pour mission de « promouvoir les intérêts professionnels du service» tandis que la seconde doit « s'efforcer de protéger les intérêts individuels et collectifs des chefs de police ${ }^{10}$.

L'association des commissaires, la Police Superintendents' Association ${ }^{11}$, représente les quelque 1500 commissaires et commissaires divisionnaires d'Angleterre et du pays de 
Galles. Les commissaires et commissaires divisionnaires écossais sont représentés par leur propre association. Etant donnée sa structure compacte et l'unité de ses membres à l'échelle nationale, la Police Superintendents' Association est extrêmement puissante, elle a sans doute davantage de poids que les deux autres associations du personnel lorsqu'il s'agit de formuler des politiques et, ce faisant, de conseiller le gouvernement. Telle n'est toutefois pas sa fonction première, si ce n'est dans le sens très général que l'association est concernée par «tout ce qui touche aux responsabilités... des membres des services de police ». Les rumeurs de fusion avec l'ACPO ou la Police Federation en raison du faible nombre de ses membres ne semblent pas fondées car l'association souhaite conserver son indépendance (bien que ses membres fassent apparemment souvent remarquer qu'ils " pourraient être intéressés par l'absorption de l'ACPO »).

Contrairement à l'ACPO, la Police Federation est avant tout une association du personnel composée de policiers allant du rang de gardien de la paix à celui d'inspecteur principal, soit 116000 membres. A la suite des grèves de 1919 dans la police, la loi sur la police (Police Act) de cette même année a institué des « fédérations de la police » pour l'Angleterre et le Pays de Galle, d'une part, et pour l'Écosse, d'autre part, dont tous les fonctionnaires de police ayant au moins rang de Chief Inspector seraient automatiquement membres. L'objectif de la Commission Desborough, qui avait élaboré les grandes lignes de la loi, était de «désyndicaliser» la police en frappant d'illégalité l'appartenance à un syndicat. Pour compenser la perte de ce droit et de celui de faire grève était créée cette Fédération de la police «dans le dessein de permettre aux membres des forces de police d'attirer l'attention des forces de police et du Secrétaire d'État sur toutes les matières affectant leurs conditions d'existence (welfare) et leur efficacité... » (Section I, Police Act, 1919). En référence à la constitution récente du Parti Travailliste et de la confédération des syndicats (Trade Union Congress), cette Fédération devait être dépourvue de tout lien "de dépendance ou d'association avec un organisme ou un individu étrangers aux services de police». A chaque unité de police correspondrait une "section » de la Fédération, comportant chacune trois comités (boards), respectivement pour les gardiens de la paix (constables), les sergents (sergeants) et les inspecteurs, y compris les inspecteurs principaux (Chief Inspectors). Les membres de ces comités de section élisaient des représentants à une conférence centrale (Central Conference) de leur grade et chacune de ces trois conférences élisait une commission centrale (Central Committee), chacune de six membres. Ces trois commissions centrales pouvaient se réunir en une commission centrale conjointe (Joint Central Committee) et les comités de section pouvaient se réunir en un comité conjoint de section (Joint Branch Board) de façon à négocier plus efficacement au nom de leurs membres. Notons qu'à l'époque la proportion entre inspecteurs et sergents était d'environ 1 à 5 et entre sergents et gardiens, de 1 à 8 . Comme chaque grade disposait et dispose toujours d'une représentation égale au sein de la commission centrale conjointe, un inspecteur avait un poids quarante fois supérieur à celui d'un gardien. Ce qui devait permettre à la hiérarchie de contrôler les gardiens, éventuellement moins fiables, et d'avoir la haute main sur l'organisation. La loi de police (Police Act) de 1970 a créé une Fédération pour les membres de la police de l'Ulster (Royal Ulster Constabulary).

Ce n'est pas sans raison que nous dressons un tableau aussi détaillé des différents services de police du Royaume-Uni. A l'échelle européenne, tout laisse à penser que l'absence d'une force de police britannique nationale s'est traduite par l'incapacité du Royaume-Uni à parler d'une seule voix et de manière convaincante. Pour résumer, il n'existe pas d'entité unique que l'on pourrait qualifier de " police britannique » mais 
un ensemble plutôt éclectique de forces de police de différentes tailles dirigées individuellement par des directeurs de police décrits comme étant "formés à s'enorgueillir de leur autonomie $\aleph^{12}$. Ils considèrent que leur rôle principal est de représenter et défendre les intérêts de leurs forces locales et on ne leur demande pas vraiment de tenir compte des besoins des services de police du Royaume-Uni au sens large.

19 Après avoir retracé le développement du système policier en Grande-Bretagne, cette étude aborde maintenant la croissance des structures chargées de la coopération transfrontalière entre services de police nationaux, avant de conclure en analysant les répercussions de l'européanisation de l'action policière sur la police britannique.

La police britannique et des structures de coopération transfrontalière

20 Au XIXème siècle, alors que l'Irlande était sous l'autorité britannique, George Bernard Shaw a qualifié de "triple contradiction " une proposition de création d'une "Royal Hibernian Academy » à Dublin. J'espère qu'il me pardonnera d'employer la même expression pour décrire la «coopération policière européenne ».

21 La coopération policière européenne est à l'ordre du jour des responsables politiques depuis plus d'un siècle ${ }^{13}$. Pourtant, les efforts pour mettre en place une police à l'échelle internationale sont décousus, improvisés et aucune méthode facilitant la coopération policière transfrontalière de manière exhaustive n'a encore été trouvée en Europe. Jusqu'à présent, les gouvernements nationaux n'ont pas eu la volonté de mettre en place à l'échelle intergouvernementale un équivalent international de "l'autorité centrale» décrite plus haut par Sir John Anderson et il faut dire que, jusqu'à récemment, il y a eu peu de raisons d'agir en ce sens. Entre temps, l'accent a été mis sur la coopération entre les différents services de police d'Europe. Ce n'est pas aussi facile qu'on pourrait le penser.

22 Par exemple, comme l'a fait remarquer Benyon, il existe au sein de l'Union européenne au moins 105 organismes identifiables qui ont des fonctions et des pouvoirs de maintien de l'ordre ${ }^{14}$ et Sheptycki fait remarquer qu'il se pourrait même qu'il y en ait plus $^{15}$. Ces organismes sont tous au service d'un système de justice pénale différent et font respecter un arsenal juridique national particulier, employant des règlements internes et des méthodes de travail spécifiques. Ils ont tous une culture, une histoire et une tradition de maintien de l'ordre différentes et rares sont ceux qui ont des normes, des procédures ou des rôles communs. En gros, on peut dire que les forces de police d'Europe sont militaires ou civiles, armées ou non-armées, judiciaires ou de maintien de l'ordre public, nationales ou locales, bien ou mal payées, et qu'elles sont un instrument soit de l'État soit des gens qu'elles servent. C'est dans ces circonstances compliquées que la coopération policière européenne a dû se développer.

23 Cette complexité, associée à l'absence (jusqu'à récemment) d'intérêt politique, a conduit à la création d'un grand nombre d'organisations de coopération dirigées pour la plupart par des hommes de terrain. Walker a décrit cette situation comme une combinaison :

24 «(...) d'agences avec des mandats, allégeances politiques, philosophies de coopération policière différents... coexistant toutes selon des rapports qui, au mieux, se traduisent par une aide et des compromis mutuels improvisés et, au pire, se transforment en concurrence ouverte sur les... ressources $»^{16}$. 

de police ou accords bilatéraux internationaux. Entre autres petites structures on peut citer l'unité de liaison européenne (European Liaison Unit - ELU) de la police du Kent, la section de liaison européenne des renseignements généraux (Special Branch European Liaison Section - SBELS) de la Metropolitan Police de Londres, le groupe de travail de la police sur le terrorisme (Police Working Group on Terrorism - PWGT), l'association européenne des polices chargées de aéroports et des ports (European Association of Airport and Seaport Police - EAASP), le «colloque des polices ferroviaires » (Colpofer) du réseau de la police européenne des chemins de fer et NEBEDEAC-Pol, l'association néerlandaise, belge et allemande des officiers de police de grade supérieur. Toutes ces structures ont en commun d'avoir été créées par des policiers de terrain en réponse à des pressions opérationnelles fonctionnelles liées au désintérêt des gouvernements.

Je me pencherai uniquement sur deux petites organisations. La première est la section de liaison européenne des renseignements généraux (SBELS) qui a été créée en 1975. Nous verrons plus tard qu'à l'époque, Interpol, alors la seule organisation policière internationale, ne s'occupait pas de terrorisme et, suite à la vague de terrorisme international déclenchée notamment par la Faction Armée rouge et Carlos « le chacal », les forces policières d'Europe ont eu besoin d'un mécanisme de coopération. En l'absence d'initiatives politiques a été créé un réseau dont les bureaux pour le Royaume-Uni étaient ceux de la SBELS. Les hommes politiques européens s'étant également rendus compte de la nécessité de trouver une réponse au problème, ils ont instauré au niveau politique le groupe de Trevi. Celui-ci a alors remplacé le réseau policier existant, ce qui a conduit la police à créer un organisme parallèle, le Police Working group on Terrorism (PWGT), pour prendre ses distances avec les responsables politiques. Ce groupe de travail existe toujours mais, comme nous le verrons plus loin, il commence à être concurrencé par Europol.

Le schéma est le même pour la seconde organisation que je voudrais évoquer, à savoir l'unité de liaison européenne (ELU) de la police du Kent qui est installée à Cheriton, à l'entrée du tunnel sous la Manche. Compte tenu de l'intensification des déplacements de personnes en Europe et de l'existence du tunnel sous la Manche, la police du Kent a ressenti le besoin pratique de coopérer avec ses homologues étrangers. Le gouvernement ayant été incapable de la conseiller, la police du Kent a été obligée de trouver ses propres solutions et c'est dans cette intention qu'elle a créé l'ELU. Cette unité est composée d'environ huit personnes et s'est taillé une réputation internationale d'efficacité.

Les grandes structures auxquelles j'ai fait référence plus haut sont notamment l'ancien groupe de Trevi (aujourd'hui dissous), Interpol, Schengen et Europol, Interpol étant sans doute la plus importante d'entre elles (un collègue allemand l'a un jour qualifiée d' « arrière-grand-mère » de toutes les organisations internationales de police). J'aimerais m'attarder un peu sur cette organisation en raison de son influence et, comme nous allons le voir, de ses défauts structurels qui ont conduit au développement d'organismes parallèles.

29 Les origines d'Interpol remontent à 1914, année au cours de laquelle le Prince Albert Ier de Monaco a été victime d'un délit dont les auteurs se sont enfuis en France. La police monégasque n'étant pas en mesure de régler cette affaire, le Prince a organisé le premier congrès international de la police à Monaco. Pour ce faire, au lieu de suivre le protocole établi et de passer par les voies gouvernementales et diplomatiques, il a 
rassemblé un groupe éclectique d'officiers de police, de juristes, de magistrats et d'autres personnes intéressées issus de quatorze pays différents qui ont été contactés quasiment au hasard (notamment par des publicités dans les journaux). Cette précision est particulièrement importante compte tenu de l'évolution ultérieure d'Interpol car, ce faisant, le Prince Albert a imprimé une direction à l'organisation, ne la considérant comme rien de plus qu'un club informel d'institutions policières, ce que, dans le fond, Interpol est restée. La Première Guerre mondiale a entravé son développement et l'organisation a repris ses activités à Vienne en 1923.

Interpol facilite aujourd'hui la coopération policière internationale en fournissant un circuit de communication entre les forces de police et les institutions pénales dans 177 pays différents ${ }^{17}$. N'ayant aucun rôle opérationnel, l'organisation a été décrite comme étant la plus fabuleuse boîte aux lettres policière du monde ${ }^{18}$.

Comme évoqué plus haut, en dépit de sa longue histoire et malgré le soutien total du gouvernement du Royaume-Uni en 1990, Interpol n'a pas été prise pour modèle de la future coopération des polices d'Europe ${ }^{19}$. Pourquoi cela? Premièrement, le statut juridique d'Interpol n'est pas clair. Cette organisation ne repose pas sur un traité ou une convention conclus à l'échelle internationale mais a été fondée par des agences de police pour répondre de manière fonctionnelle à un besoin opérationnel donné. Selon Anderson, seules cinq entités ont officiellement reconnu Interpol : l'ONU, le Conseil de l'Europe, la France, la Thailande et les Etats-Unis, ses autres participants lui accordant ce qu'il appelle « une reconnaissance tacite $»^{20}$. De plus, les relations entre Interpol et l'Organisation des Nations unies sont confuses. Interpol a été officiellement placée dans la catégorie des organisations non gouvernementales en 1949 mais elle a été " promue » en 1971, de sorte que dans certaines situations elle est considérée comme une organisation intergouvernementale ${ }^{21}$. Néanmoins, en 1998 l'Assemblée générale de l'ONU a accordé à Interpol le statut d'observateur selon le principe inexpliqué qu'elle était désormais reconnue comme une organisation intergouvernementale.

Deuxièmement, Anderson ${ }^{22}$ a fait remarquer que la constitution d'Interpol qui a été rédigée en 1956, non pas par des juristes internationaux mais par des officiers de police, est dépassée et qu'elle "n'est pas bien formulée ». Par exemple, l'article 4 stipule que «tout pays peut déléguer comme membre de l'organisation tout organisme de police officiel... ». Le fait que les membres d'Interpol soient des organismes de police et non des "pays» s'explique par la volonté de séparer la politique nationale des fonctions d'Interpol. Cette indépendance fondamentale par rapport à tout contrôle politique est profondément ancrée dans l'histoire d'Interpol et sera probablement impossible à changer. L'article 14 prévoit que les décisions de modification de la constitution doivent être prises à la majorité des deux tiers des membres. Comme tous les membres ont les mêmes droits de vote et comme, pour toutes sortes de raisons, environ $20 \%$ des membres ne participent pas aux assemblées générales, il est extrêmement difficile d'introduire des changements. De plus, absolument aucune procédure d'expulsion des membres d'Interpol n'est prévue. C'est pour cette raison que Cuba, qui n'a jamais payé ses droits d'adhésion, est néanmoins membre d'Interpol ${ }^{23}$.

33 Troisièmement, et c'est peut-être le principal problème, l'article 3 de la constitution d'Interpol lui interdit de s'occuper de questions de nature « politique, militaire, raciale ou religieuse » et, par le passé, Interpol a inclus l'immigration, le terrorisme et le nazisme ${ }^{24}$ dans cette définition. Malgré les explications de certains commentateurs ${ }^{25}$, Interpol est restée fidèle à ce principe jusqu'à une période relativement récente, ce qui 
a considérablement nui à sa réputation. Par exemple, jusqu'à la publication, en 1986, d'une "notice rouge" pour Josef Mengele, Interpol avait toujours refusé d'aider à rechercher les criminels de guerre nazis. Marcel Sicot, un ancien secrétaire général de l'organisation, a défendu l'article 3 dans un écrit datant de 1961 en indiquant que la recherche systématique des criminels nazis obligerait Interpol à intervenir dans de nombreux autres cas du même type ${ }^{26}$. De la même façon, suite aux actes terroristes contre les athlètes israéliens aux Jeux olympiques de Munich de 1972, Interpol, par décision de son secrétaire général, Jean Népote, refusa d'aider la police allemande dans son enquête. Il s'ensuivit un débat qui conduisit à la décision de clarifier la position d'Interpol concernant l'article 3, ce qui s'est finalement traduit par le vote d'une résolution en 1984. Malgré cela, l'aide d'Interpol dans les affaires terroristes ne peut être garantie car le secrétariat général peut toujours invoquer l'article 3. Cet Etat de fait a contribué à la création du groupe de Trevi en 1975.

Par ailleurs, Interpol n'étant pas disposée à intervenir dans les aspects administratifs des questions d'immigration, cela a compliqué la tâche des architectes de l'accord de Schengen car ils avaient besoin d'Interpol pour remédier à la suppression des frontières intérieures. C'est pourquoi, lorsque le système d'information de Schengen a été mis en place, sa base de données a été conçue selon le modèle du dispositif de recherche automatique ASF (Automated Search Facility) d'Interpol, augmenté de la base de données des personnes qui se sont vu refuser l'entrée en Europe aux frontières extérieures de Schengen.

Il faut dire que les officiers de police n'ont pas une haute opinion d'Interpol et leurs commentaires sur la qualité, la sécurité et la rapidité de ses fonctions sont rarement élogieux. Fijnaut a parlé de "l'organisation bureaucratique, globale, inefficace d'Interpol »" Woodward ${ }^{28}$ a cité l'observation selon laquelle « de nombreux officiers de police préféreraient transmettre leurs informations opérationnelles à Interflora plutôt qu'à Interpol ». Benyon a écrit que «les longs retards et l'insécurité générale [d'Interpol] viennent compléter une mise en accusation implacable $»^{29}$. Tout en recommandant le développement d'Interpol pour en faire la nouvelle agence européenne de police, le British Home Affairs Committee (comité britannique des affaires intérieures) a fait savoir qu'il avait des doutes sur Interpol concernant «sa vitesse de réaction, la rigidité de ses procédures et ses faibles niveaux de sécurité $»^{30}$.

Enfin, Interpol n'a aucune responsabilité juridique ou constitutionnelle envers le public. Par exemple, bien qu'elle soit financée par les contribuables, ses comptes financiers ne sont fournis qu'aux gouvernements membres, ils ne peuvent être mis à la disposition directe du public. Bien qu'elle traite des données informatisées, certains ont dit qu'Interpol présentait peu de garanties en matière de protection des données ${ }^{31}$. A l'heure actuelle, chaque BCN (bureau central national - le bureau d'Interpol dans chaque pays) est tenu de respecter la législation sur la protection des données en vigueur dans son pays, s'il en existe une. Peu de pays sont effectivement en mesure de contrôler l'utilisation des informations fournies qui sera faite dans d'autres pays. Interpol reconnaît d'ailleurs que cela pose problème, ce qui a conduit la 20ème Conférence régionale européenne à créer un groupe de travail pour examiner la question de la protection des données. Le groupe a étudié plusieurs options pour l'avenir et a conclu que l'adoption d'une convention signée à l'échelle internationale et ayant force exécutoire «serait la meilleure solution à tous les problèmes que nous rencontrons actuellement lors des échanges d'information passant par Interpol ». Le groupe a pleinement 
conscience de la difficulté, voire la quasi-impossibilité, d'agir en ce sens et a indiqué qu'il ne fallait pas s'attendre à ce que cette mesure se concrétise "dans la prochaine décennie $»^{32}$. En contrepartie, lors de l'assemblée générale de Rome, en 1994, Interpol a pris une résolution visant à obliger ses membres à respecter toutes les conditions d'utilisation de ces informations imposées par les États émetteurs.

La seconde évolution majeure en matière de maintien de l'ordre à l'échelle européenne est le groupe de Trevi. Interpol étant peu disposée à s'occuper de terrorisme, en décembre 1975 le Conseil des ministres a mis en place le groupe de Trevi dans le cadre de la Coopération politique européenne intergouvernementale en lui donnant pour mission d'examiner les possibilités de coopération policière européenne, en particulier en matière de terrorisme. Le groupe de Trevi était organisé selon une hiérarchie à trois niveaux : le niveau ministériel, celui des hauts représentants gouvernementaux et enfin celui des groupes de travail.

Le groupe s'est réuni pour la première fois en juin 1976 à Rome et le mystère entourant ce groupe est tel que même l'origine de son nom est floue et invérifiable. Le groupe a très probablement pris le nom des fontaines de Trevi qui se trouvent près de l'endroit où s'est déroulée la première conférence ${ }^{33}$. Les cinq groupes de travail suivants ont été créés le 31 mai 1977 après la réunion des ministres de l'Intérieur de la CE de 1976 qui s'est tenue au Luxembourg et c'est le groupe de Trevi qui a adopté la section de liaison européenne des renseignements généraux (SBELS) dont il a été question plus haut.

La troisième évolution majeure en matière de coopération policière européenne est l'accord de Schengen suivi de la convention du même nom. Il s'agit d'initiatives menées et développées à l'échelle politique qui visent à supprimer les frontières intérieures de l'Europe. L'accord de Schengen est évoqué ici car il constitue un mécanisme de coopération policière transfrontalière rivalisant avec Interpol mais il a en fait été signé pour éviter bon nombre des problèmes rencontrés par cette organisation. Bien qu'il ait été intégré dans le champ des compétences de l'Union européenne par le traité d'Amsterdam (TA), il nous semble utile de décrire ici l'historique de l'accord de Schengen pour mesurer son influence sur la coopération policière au sein de l'Europe.

L'initiative politique Schengen s'est traduite par la signature de l'accord de Sarrebruck en 1984, qui se donnait pour objectif ultime la suppression de tout contrôle aux frontières entre la France et l'Allemagne. Le 14 juin 1985 la proposition fut élargie aux pays du Bénélux. Cet accord, officiellement dénommé « Accord concernant l'abolition graduelle des contrôles aux frontières communes conclu entre les gouvernements des Etats de l'Union économique du Bénélux, de la République Fédérale d'Allemagne et de la République française ", a été signé à bord d'une péniche amarrée sur la Moselle dans la ville luxembourgeoise de Schengen, symboliquement choisie pour sa proximité de la frontière franco-germano-luxembourgeoise.

41 Alors que Schengen n'avait été au départ guère plus qu'une déclaration d'intentions, il donna naissance en 1990 à un accord de mise en oeuvre, officiellement intitulé "Convention pour l'application de l'accord de Schengen du 14 juin 1984 sur l'abolition graduelle des contrôles aux frontières communes entre le Royaume des Pays-Bas, le Royaume de Belgique, le Grand-Duché de Luxembourg, la République Fédérale d'Allemagne et la République française ». Ce délai de cinq années était dû pour une bonne part aux changements intervenus dans le climat politique en Europe à la suite de l'effondrement des régimes communistes d'Europe de l'Est à la fin des années 1980. L'accord de 1990 donnait à l'entente initiale une certaine substance en établissant des 
règles internationalement contraignantes et est parfois désigné comme Schengen II, celui de 1985, devenant Schengen I. Les premiers signataires furent rejoints ensuite par l'Espagne, le Portugal et la Grèce, ce qui ne laissait plus à l'écart, sur les douze membres que comptait alors la Communauté Européenne, que le "trio de 1973 ", soit le Royaume-Uni, le Danemark et la République d'Irlande. Le Danemark signa à son tour l'accord en 1995, après s'être limité à un statut d'observateur en juin 1994. Les pays qui ont adhéré à l'Union Européenne en 1995 se sont tous déclarés intéressés par l'accord de Schengen et la Finlande et l'Autriche l'ont signé cette même année.

Schengen II contenait des sections sur l'immigration et le droit d'asile et une section ample et détaillée sur la coopération policière transfrontalière comportant des règles très précises sur les opérations transfrontalières et notamment le droit de suite. L'accord proposait également la création sans précédent d'un réseau d'information et de recherche et d'échange de renseignements en matière criminelle. On a pu qualifier Schengen I de gentleman's agreement par lequel des gouvernements s'attaquaient à la tâche d'abolir (sic) graduellement les contrôles à leurs frontières communes ${ }^{34}$. Si donc la raison d'être de l'accord de Schengen était la suppression des contrôles aux frontières, toutes ses clauses, notamment celles qui confèrent à la police des pouvoirs accrus en matière de droit de suite transfrontalière et celles qui prévoient l'échange de données informatiques, peuvent être considérées comme relevant purement et simplement de la suppression de frontières intérieures.

L'insistance de l'accord de Schengen sur la suppression des contrôles aux frontières implique, comme le souligne Gregory ${ }^{35}$, qu'il s'agit davantage de frontières terrestres que maritimes. Les Britanniques ont toujours accueilli au mieux avec tiédeur l'idée de supprimer les contrôles à leurs frontières et cette attitude n'était certainement pas inconnue des architectes tant de l'Acte Unique Européen que de Schengen. En tant qu'État insulaire, la Grande-Bretagne a fait valoir qu'elle voyait des avantages spécifiques à contrôler ses frontières, qui sont des frontières naturelles, à la différence des Etats qui ont de longues frontières terrestres difficiles à surveiller efficacement. Historiquement, elle a donc compté sur l'efficacité de ses contrôles frontaliers et ses services de police des frontières ont acquis une grande compétence ${ }^{36}$. Traditionnellement, elle n'a donc pas eu besoin, en plus des contrôles frontaliers, de contrôles exercés après l'entrée dans le pays ou à l'intérieur de celui-ci, qui sont courants dans les autres pays de l'Union Européenne. Ceux-ci consistent notamment dans l'obligation faite aux étrangers de se faire enregistrer auprès de la police du pays d'accueil, ce qui implique la tenue d'un registre national des étrangers, l'obligation de porter sur soi et de présenter sur réquisition d'un policier une carte d'identité et, en France, par exemple, le droit reconnu à la police d'arrêter les gens et de les détenir à seule fin d'établir leur identité. On a fait valoir que la suppression des contrôles aux frontières telle que la prévoit Schengen imposerait l'adoption de mesures compensatoires du type de celles-ci. Ce qui rencontrerait au Royaume-Uni une très vive résistance, et d'abord de la part des organismes de protection des libertés civiles qui considèrent de telles mesures comme des abus. On a également objecté que les individus qu'il serait le plus nécessaire de contrôler après le passage de la frontière, c'est-à-dire ceux qui se livrent à la contrebande et autres délits, sont précisément ceux dont on peut le moins attendre qu'ils se plient à ces règlements. La Grande-Bretagne a enfin exprimé ses doutes quant à la sécurité des frontières extérieures de Schengen, mentionnant notamment les services d'immigration italiens et les quelque 200 îles inhabitées qui se trouvent au large de la Grèce continentale et voyant là autant de 
raisons supplémentaires pour conserver ses contrôles frontaliers. En 1998, toutefois, et suite à l'intégration des dispositions de Schengen dans le dispositif de l'Union Européenne, le gouvernement britannique a annoncé que la Grande-Bretagne allait demander à adhérer partiellement à l'accord de Schengen. Cela lui donnerait accès au Système d'Information de Schengen (SIS), à l'exclusion de ce qui concerne l'immigration, et permettrait la surveillance transfrontalière. Le SIS et le système complémentaire SIRENE seront basés au sein du NCIS et il est vraisemblable que les mesures législatives nécessaires ainsi que l'infrastructure seront mises en place en 2003.

Les principales étapes de l'évolution en matière de coopération policière européenne sont exposées dans le traité sur l'Union européenne (TUE) de 1992. Le TUE a mis sur pied une nouvelle structure, l'Union européenne, ce qui, du point de vue britannique, était une formidable avancée par rapport au concept originel de marché commun européen. L'Union européenne devait s'articuler autour de trois " piliers », le troisième portant sur la justice et les affaires intérieures (JAI) et absorbant l'ancien groupe de Trevi. Le pilier JAI englobait Europol, alors à l'État embryonnaire, et qui présente un grand nombre d'avantages par rapport à Interpol. Elle se fonde sur un traité signé au niveau européen, elle a un statut juridique et est comptable envers les citoyens d'Europe sur le plan de ses activités, de ses finances et de la protection des données. Par contre, Europol est la première organisation de ce type qui n'a pas été créée par des officiers de police pour répondre à des besoins fonctionnels, ce qui explique en partie qu'elle soit si lente à se mettre en place. Il est permis de penser que sa création est intervenue trop tôt dans l'histoire de l'Union européenne et que, d'un point de vue fonctionnel, elle ne se justifiait pas encore. Nous avons déjà vu que le PWGT, qui est dirigé par la police, a souhaité rester à l'écart du groupe de Trevi, et il semblerait qu'il veuille également garder ses distances avec Europol qui a aussi pour mission de lutter contre le terrorisme.

Après avoir évoqué le développement des principaux instruments de la coopération européenne en matière policière, voyons maintenant les répercussions de l'européanisation sur la police britannique. Il y en a principalement deux. Tout d'abord, elle pousse à un certain degré de nationalisation, soulignant ainsi les faiblesses de la structure policière britannique du XIXème siècle. Ensuite, elle entraîne un certain degré de "convergence» des réponses nationales en matière de police criminelle, comme le manifestent les interactions entre les divers organismes impliqués dans l'action policière.

Le meilleur exemple de ce phénomène de nationalisation initié sous l'impulsion de l'Europe est la mise en place du service national des renseignements criminels (le $\mathrm{NCIS)}$, créé pour répondre aux demandes de coordination renforcée émanant du groupe de Trevi. Selon la théorie néofonctionnaliste, une fois en place, tout organisme de type NCIS chargé d'une fonction d'intégration dans un domaine spécifique d'activité crée une dynamique de renforcement de cette intégration. C'est ce qu'on appelle «l'effet de débordement ». Il se produit lorsqu'un organisme de ce genre repère dans des domaines voisins des champs d'intervention possibles. Le NCIS était chargé d'intégrer à l'échelon du Royaume-Uni l'analyse des renseignements en matière criminelle, mais ses activités ont « débordé » sur des domaines apparentés, tels que la coopération policière internationale, les enquêtes sur la délinquance financière et plus récemment certains aspects de la lutte anti-terroriste. 

brigade criminelle nationale, la National Crime Squad (NCS). Cette entité qui a été créée à partir des anciennes brigades criminelles régionales est la branche opérationnelle du NCIS et bénéficie d'un statut égal. En effet, elles ont toutes deux obtenu le «statut d'agence » en 1998, ce qui signifie qu'elles disposent de plus d'indépendance financière et opérationnelle qu'auparavant et qu'elles peuvent employer du personnel directement, de leur propre chef, au lieu de faire appel aux officiers des postes de police. Les directeurs généraux du NCIS et de la NCS ont le même grade qu'un directeur de police.

n du NCIS et de la NCS constitue une étape sur la voie de la nationalisation des services de police britanniques qu'il ne faut pas sous-estimer. Compte tenu de l'élargissement de leurs attributions en réaction aux pressions européennes - par exemple en prenant en charge le versant britannique de Schengen - ils pourraient avoir un impact considérable sur l'organisation de la police au Royaume-Uni en favorisant le centre au détriment des régions. Cela pourrait remettre en cause l'indépendance et l'autonomie des directeurs de police. Il y a fort à parier que la demande de forces de maintien de l'ordre transnationales va augmenter et cela incitera encore davantage à développer ce types d'organismes nationaux.

Une des meilleures indications de l'influence de l'Europe sur la police britannique est la «convergence» des différents services de police du Royaume-Uni. Par exemple, compte tenu de leur rôle, les services douaniers (HMCE) ont pris en charge la majeure partie des responsabilités de lutte contre la criminalité internationale et particulièrement contre les importations et les exportations illégales. C'est particulièrement vrai pour la drogue. Par contre, la police a la priorité en matière de lutte contre la drogue à l'intérieur du pays. Ce partage des responsabilités se reflète dans la structure du NCIS et de l'organisme auquel il a succédé, le National Drugs Intelligence Unit (office central pour la répression du trafic illicite des stupéfiants). Etant donné que, outre leur rôle traditionnel et pour refléter l'évolution de l'époque à laquelle nous vivons, la primauté de la lutte contre le grand banditisme international a été récemment accordée aux services secrets de renseignement, nous pouvons constater qu'en plus de la police deux agences séparées s'occupent désormais de criminalité internationale. Le terrorisme et l'immigration illégale font maintenant partie des attributions d'Europol et, ce faisant, le Security Service (qui a la priorité en matière de lutte contre le terrorisme, en association avec les renseignements généraux) et le service d'immigration du Royaume-Uni (UKIS) travaillent également en partenariat avec le NCIS. Celui-ci recouvre donc cinq institutions différentes souvent amenées à défendre les intérêts les unes des autres. Un exemple concret: beaucoup d'officiers de liaison britanniques spécialisés dans la lutte contre la drogue en poste à l'étranger sont des officiers du HMCE. Ils peuvent être amenés à accomplir les diverses activités qui sont du ressort du NCIS et il n'est donc pas rare qu'ils interviennent dans toutes sortes d'affaires criminelles (notamment les meurtres) pour lesquelles le HMCE n'est traditionnellement pas compétent. En outre, pour ce qui est de la supervision et de la gestion, un HMCE Supervisor peut avoir la responsabilité des activités de police du personnel de l'UKIS opérant dans son équipe et vice-versa. Bien qu'à court terme cette façon de procéder puisse être synonyme d'inefficacité, de factionalisation et de confusion, à long terme elle pourrait tout aussi bien devenir synonyme 
d'harmonisation, d'ordre et de convergence. Se pourrait-il vraiment que l'unification des forces de police dépasse les limites du possible?

Qu'en est-il donc de l'avenir? C'est dans la police des frontières du Royaume-Uni que l'unification est la plus probable. A l'heure actuelle, trois agences différentes sont concernées: le HMCE, l'UKIS et la police représentée par des officiers des renseignements généraux qui traitent principalement de la législation sur le terrorisme. Les trois agences peuvent très bien se remplacer les unes les autres, en particulier la police qui a des pouvoirs plus étendus que les autres agences. Depuis que, récemment, le Royaume-Uni a demandé à s'associer à certaines parties de l'accord de Schengen, un service frontalier unifié pourrait être mis en place.

Etant donnés les délais d'application de la coordination fixés par le traité d'Amsterdam et la coopération accrue entre les autorités judiciaires européennes compte tenu de l'harmonisation des systèmes européens de justice pénale, je pense qu'à l'avenir le CPS (le Ministère public) tiendra un rôle plus important et sera plus influent, en particulier dans sa manière d'orienter la police dans ses enquêtes. Je pense également que le NCIS et la NCS joueront un rôle accru et que les liens se développeront avec Europol, laquelle deviendra encore plus puissante. Je conçois aisément que les renseignements généraux puissent devenir un organisme national indépendant ou faisant partie du NCIS mais j'ai plus de mal à imaginer que l'État britannique puisse pour l'instant fermer les services de sécurité ou les services secrets de renseignement. Je ne pense pas que cela se produise dans un proche avenir. L'Etat britannique est on ne peut plus prudent, en particulier dès que le mot "Europe " est prononcé. Pour conclure, j'aimerais proposer une autre citation extraite du discours de Sir John Anderson et attirer tout particulièrement l'attention sur le dernier mot: "Il me semble que le système policier de ce pays, et c'est une caractéristique commune à la plupart des grandes institutions de Grande-Bretagne, est le résultat du processus d'évolution graduelle $»^{37}$.

\section{NOTES}

1. . Anonyme, « Police Co-operation and Co-ordination. Report of Sir John Anderson's speech to the 1923 meeting of the Chief Constables Association », The Police Review and Parade Room Gossip, Londres, 8 June 1923, pp.298-299.

2. . Cet article est issu d'une communication présentée au séminaire «Questions de Polices/Policing Matters », Gern, Paris 23-24 mars 2001), organisé par Jean-Marc Berlière, Clive Emsley, René Lévy et Dominique Monjardet). Texte traduit de l'anglais, relu par René Lévy.

3. . ACPO, Secretariat International, National and Inter-Force Crime (The Phillips Report), A study commissioned by ACPO, Londres, Unpublished, 1996.

4. . Le United Kingdom Immigration Service était appelé jusqu'à la fin des années 1990 Her Majesty's Immigration Service. On prétend que ce changement d'appellation est 
survenu lorsqu'on eut découvert que ce service n'avait pas le droit d'utiliser le nom du monarque dans son intitulé.

5. . D'ordinaire, les policiers en tenue sur la voie publique ne sont pas armés, mais disposent d'un ensemble d'équipements défensifs incluant un bâton télescopique (qui a largement remplacé la matraque traditionnelle), des menottes et une bombe de gaz CS. Dans la plupart des services, les seuls policiers qui soient régulièrement armés sont les membres de l'unité des armes à feu chargés de quatre missions : la garde des sites sensibles (comme les aéroports) ; la protection des personnalités ; les rondes de soutien aux policiers sans armes, lorsqu'il s'agit de réagir à des incidents impliquant des armes à feu ; et enfin les interventions comportant de telles armes. Les enquêteurs, pour leur part, ne sont autorisés à porter d'armes que pour des raisons particulières, à condition d'être entraînés à leur usage et d'y être autorisés par un officier supérieur.

6. . Reiner R., "Where the Buck Stops : Chief Constables Views on Police Accountability ", in Morgan R.Smith, D. (Eds.), Coming to terms with policing, Londres, Routledge, 1989, pp. 208-209.

7. . Il est intéressant de noter que le gouvernement a alloué un budget d'environ 25 millions de livres sterling au NCIS, ce qui lui a permis de louer des bureaux dans un complexe industriel de Lambeth. Dans le même temps, des bureaux ont été conçus sur mesure à l'intention des services secrets de renseignement à moins de 400 mètres du siège social du NCIS. Les coûts se sont élevés à 283 millions de livres sterling.

8. . L'ACPO remplace le County Chief Constables Club, une organisation représentant les directeurs des forces de police des comtés qui a été créée en 1858 et à laquelle s'est associée en 1896 une association représentant les directeurs des forces de police urbaines et municipales d'Angleterre et du pays de Galles, la Chief Constables Association of England and Wales. Entre 1910 et 1920 ont été organisées un certain nombre de conférences régionales présidées par des représentants du ministère de l'Intérieur et visant à améliorer la coopération entre le gouvernement et les officiers de police. Le pays était divisé en huit régions (ce découpage existe toujours) et chaque directeur de police était membre de sa conférence locale. En 1918 a été mise sur pied une conférence centrale destinée à coordonner le travail des conférences régionales. Jusqu'en 1922, les conférences avaient pour objectifs « de rapprocher les forces de police du ministère de l'Intérieur, d'améliorer le contact entre les ministères et la police partout en Angleterre et au pays de Galles et, enfin, d'aider le ministère de l'Intérieur à donner les instructions éventuellement requises pour guider la police » (ibid.). Les deux organisations ont fusionné en 1948 à l'instigation du gouvernement avant d'être rejointes en 1970 par la Royal Ulster Constabulary (la police d'Irlande du Nord), formant ainsi l'Association of Chief Police Officers of England, Wales and Northern Ireland, ou ACPO.

9. . Swallow P., European Police Cooperation : A Comparative analysis of European level Institutional and organisational developments and national level polices and structures, PhD thesis, University of Southampton, Unpublished, 1998, en particulier pp. 131-136.

10. . Barton M., Double vision, Policing Today, 1996, 2, 1, pp. 8-9.

11. . La Police Superintendents' Association a été créée en 1920 pour coordonner la représentation des commissaires au Police Council (conseil de la police) où étaient requis des représentants de tous les rangs de la police. L'association s'est développée, le nombre de membres a augmenté et, en 1963, elle a pu bénéficier d'une aide à plein temps pour les travaux de secrétariat. En 1977 lui a été détaché à plein temps un 
officier de police financé par l'autorité centrale, le secrétaire national. L'association emploie aujourd'hui six personnes à temps complet : le secrétaire national, son adjoint, le président et trois agents administratifs. De surcroît, elle a fait l'acquisition de ses propres locaux dans le Berkshire. Au début des années 90 a été mis en place un système de comités s'inspirant ouvertement du système de comités de l'ACPO. L'association s'est efforcée de conserver son autonomie par rapport à la Police Federation, qui est beaucoup plus grande, et à l'ACPO, qui jouit d'une autorité plus importante, et elle souhaite user de son influence en toute indépendance.

12. . Reiner R., «Where the Buck Stops : Chief constables Views on Police Accountability ", in Morgan, R.Smith, D. (Eds.), Coming to terms with policing, Londres, Routledge, 1989, p. 215.

13. . Anderson M., Den Boer (Eds.), Policing Across National Boundaries, Londres, Pinter 1994, introduction.

14. . Benyon J., Turnbull L. et al., Police Cooperation in Europe : An Investigation, Leicester, CSPO, 1993, chap. 3.

15. . Sheptycki J.W.E., « Transnational Policing and the Makings of the Postmodern State ", British Journal of Criminology, 1995, 35, 4, p. 616.

16. . Walker N., «The International Dimension », in Reiner R. et Spencer M., (Eds.), Accountable Policing, Londres, Institute for Public Policy Research, 1993, p. 125.

17. . Le chiffre varie presque toutes les semaines en fonction du climat politique international. Parmi les pays ayant récemment rejoint Interpol on peut citer l'Afrique du Sud, le Salvador, la République tchèque, la Slovaquie, la Biélorussie et la Macédoine. Le $\mathrm{BCN}$ de Londres a publié une liste des douze ou treize pays identifiés comme n'étant pas membres de l'organisation en 1994. Il s'agissait de l'Afghanistan, du Bhoutan, des Comores, du Laos, de la Corée du Nord, de l'île Pitcairn, des îles Salomon, du Yémen-duSud, de Taiwan, de Tuvalu, de Vanuatu, des Samoa-Occidentales et on pourrait également ajouter la Yougoslavie.

18. . Greilsamer L., Interpol - le Siège du Soupçon, Paris, Alain Moreau, 1986, introduction.

19. . Pour en savoir plus sur le rejet d'Interpol en tant qu'agence européenne de police, se reporter Swallow P., « Of limited Operational Relevance : A European View of Interpol's Crime fighting Role in the Twenty-First Century ", Transnational Organised Crime, 1996, 2, 4, pp.106-130.

20. . Anderson M., Policing the World : Interpol and the Politics of International Police Cooperation, Oxford, Clarendon Press, 1989, p. 71.

21. . United Nations, Special arrangement for co-operation between the United Nations and the International Criminal Police Organisation Interpol, UNESCO Resolution E/RES/ 1579 L 20 May 1971, New York, UNO 1971.

22. . Anderson M., Policing the World : Interpol and the Politics of International Police Cooperation, Oxford, Clarendon Press, 1989, p. 182.

23. Certaines sanctions limitées, telles que la suppression du droit de vote aux assemblées générales, peuvent être appliquées en vertu de la réglementation générale 52. Toutefois, l'ancien président d'Interpol, Norman Inkster, a reconnu que « les dispositions de l'article 52 sont inefficaces » (ICPR $n^{\circ} 451: 3$ ).

24. . Pour en savoir plus sur le supposé soutien tacite des nazis par Interpol, se reporter à Garrison O., The Secret World of Interpol, McLellan, 1977.

25. . Par exemple, Babovic B., Interpol face au terrorisme, Belgrade, 1989.

26. . Greilsamer L., Interpol, le Siège du Soupçon, Paris, Alain Moreau, 1986, p. 92. 
27. . Fijnaut C., « Policing Western Europe : Interpol, TREVI and Europol », Police Studies, 1992, 15, 2, p. 101.

28. . Woodward R., The Establishment of Europol : A Critique Paper, presented to the Cyprus Police Academy International Seminar, Nicosia, 20/22 April 1993, Leicester, 1993, p. 3.

29. . Benyon J., Turnbull L., et al., Police Cooperation in Europe : An Investigation, Leicester : CSPO, 1993, p. 223.

30. . Home Affairs Committee, Practical Police Cooperation in the European Community, Session 1989-90 : 363 - II (Vols. I and II), Londres, HMSO 1990, vol. 1, p. 25. 31. . Grange, K., The Impact of Data Protection Upon European Police Information Flows, Paper given at the European Consortium for Political Research, Madrid 17 April 1994 (Unpublished).

32. . Interpol European Working Party on Data Protection, Draft Report to the European Regional Conference, Lyons, Unpublished, 1993, p. 22.

33. . Selon Bresler, le ministre de l'Intérieur néerlandais, M. Fontain, qui présidait la première réunion du groupe, aurait vu la fontaine de Trevi en regardant par la fenêtre et dit : « appelons-le 'Trevi' et ils furent tous d'accord ». Malheureusement, Bresler ne cite pas ses sources (Bresler, F., Interpol, Londres, Mandarin, 1993).

34. . Meijers H., et al., Schengen : Internationalisation of the Central Chapters of the Law on Aliens, Refugees, Security and the Police, Zwolle/Deventer, W.E.J. Tjeenk Willink,1991, p.1.

35. . Gregory F.E.C., Image and Reality in British Border Control Policy 1988-1993, Paper presented to the ECPR conference, Madrid, Unpublished, 1994, p.7.

36. . Gregory F.E.C., The Schengen Agreements - Reactions in a Non-participating State. The Case of the UK, Paper given to the CERI Conference « La Table Ronde sur les Accords de Schengen », Paris, Unpublished, 1991.

37. . Anonyme, « Police Co-operation and Co-ordination. Report of Sir John Anderson's speech to the 1923 meeting of the Chief Constables Association », The Police Review and Parade Room Gossip, 8 June 1923, p. 298.

\section{RÉSUMÉS}

Cet article décrit le rôle des pouvoirs locaux dans la croissance du système policier britannique depuis le début du 19e siècle jusqu'à nos jours. Il examine l'introduction d'organes de coordination et de représentation nationaux et décrit leur rôle actuel. Du point de vue britannique, il analyse ensuite le développement des organisations internationales visant à faciliter et coordonner les activités policières transfrontalières européennes. Prenant pour point de départ la création d'Interpol au début du 20e siècle, il examine les problèmes relatifs à cette organisation, qui ont conduit à la mise en place ultérieure d'organismes tels que le Groupe Trévi ou Europol. Finalement, l'article se penche sur l'influence que l'"européanisation" croissante de l'activité policière exerce sur la police britannique, et en particulier le rôle grandissant d'organismes nationaux et la "convergence" qui rapproche des institutions policières ayant des missions voisines. 
This paper describes the parochially led growth of the British policing system from its beginnings in the early part of the 19th century until the present day. It discusses the inception of police coordinating and representative bodies at the national level, and outlines their respective present-day roles. This is followed by a description from a British perspective of the development of international organisations intended to facilitate and coordinate cross border police activities within Europe. Starting with Interpol in the early part of the 20th century, the paper addresses the problems inherent in that organisation which led to the establishment of later bodies such as the Trevi Group and Europol. Finally the paper links these two sections by examining the influences that increasing Europeanisation of policing is having upon the British police service, particularly the increasing importance of national bodies and the 'convergence' that is taking place between policing bodies with similar roles.

INDEX

Index géographique : Europe, Royaume Uni

Mots-clés: Gendarmeries et maintien de l'ordre, police 